\title{
Calidad de la formación del comunicador en tiempos de pandemia
}

\author{
Quality of communicator training in times of pandemic
}

Qualidade da formação de comunicadores em tempos de pandemia

\section{ARTÍCULO GENERAL}

\section{Cusipuma Arteaga Rosa \\ https://orcid.org/0000-0002-1088-3025 \\ rcusipumaa@unmsm.edu.pe \\ Universidad Nacional Mayor de San Marcos, Lima, Perú}

Recibido 25 de Diciembre 2021 | Arbitrado y aceptado 25 de Diciembre 2021 | Publicado el 28 de Enero 2022

\section{RESUMEN}

Los medios de comunicación fueron introducidos en la escuela para colaborar en el proceso educativo. Si bien es cierto, lo que se buscaba en un inicio era traspasar las paredes del aula para llevar a los estudiantes al encuentro de realidades astronómicas como el espacio o invisibles al ojo humano como las células; ya antes habían sido utilizadas en la capacitación de campesinos, mineros y organizaciones de base en América Latina, en particular, desde mediados del S.XX. El desarrollo de la tecnología ha permitido que hoy los medios tradicionales se encuentren presentes en el ciberespacio, se encuentren en internet facilitando la educación de niños y jóvenes, como parte de la infraestructura que asegura estándares mínimos de calidad en nuestros sistemas educativos.

En este contexto, la formación de los nuevos profesionales se ha visto influenciada por la presencia de las tecnologías, más aún debido a las condiciones de aislamiento impuestas por la pandemia de salud del COVID-19. Así, hoy en día, la formación de los futuros comunicadores sociales demanda que éstos no solamente dominen las técnicas de redacción de los distintos géneros informativos, también deben sacar provecho de los recursos de internet y programas informáticos que les faciliten la presentación de datos, gráficos, tablas, conocer el lenguaje de los medios y las técnicas para colocar mensajes en los distintos entornos de comunicación y las redes sociales. Adicionalmente, planificar y llevar a efecto proyectos de comunicación de diferente índole para ser aplicados en diferentes contextos y poblaciones.

El presente artículo muestra el mundo de posibilidades ofrecidas por internet para la formación de los comunicadores sociales quienes a su vez, capacitaron a distintas poblaciones del territorio nacional durante el primer año de la pandemia mientras realizaban sus estudios en la modalidad remota.

Palabras clave: Comunicación Educativa; Educomunicación; Comunicación mediada; materiales multimediales; videos tutoriales

\section{ABSTRACT}

The media were introduced in the school to collaborate in the educational process. Although it is true, what was sought at the beginning was to go beyond the walls of the classroom to take students to meet astronomical realities such as space or invisible to the human eye such as cells; They had already been used before in the training of peasants, miners and grassroots organizations in Latin America, in particular, since the middle of the 20th century. The development of technology has allowed traditional media to be present in cyberspace today, to be found on the Internet, facilitating the education of children and young people, as part of the infrastructure that ensures minimum quality standards in our educational systems.

In this context, the training of new professionals has been influenced by the presence of technology, even more so due to the isolation conditions imposed by the COVID-19 health pandemic. Thus, today, the training of future social communicators demands that they not only master the writing techniques of the different news genres, they must also take advantage of Internet resources and computer programs that facilitate the presentation of data, graphics , tables, knowing the language of the media and the techniques to place messages in the different communication environments and social networks. Additionally, plan and carry out communication projects of a different nature to be applied in different contexts and populations.

This article shows the world of possibilities offered by the internet for the training of social communicators who, in turn, trained different populations of the national territory during the first year of the pandemic while they were carrying out their studies remotely.

Keywords: Educational Communication; Educommunication; mediated communication; multimedia materials; tutorial videos

\section{RESUMO}

As mídias foram introduzidas na escola para colaborar no processo educacional. Embora seja verdade, o que se buscava no início era ir além das paredes da sala de aula para levar os alunos a conhecer realidades astronômicas como o espaço ou invisíveis ao olho humano como as células; Eles já haviam sido usados antes na formação de camponeses, mineiros e organizações de base na América Latina, em particular, desde meados do século XX. O desenvolvimento da tecnologia permitiu que a mídia tradicional estivesse presente no ciberespaço hoje, encontrada na Internet, facilitando a educação de crianças e jovens, como parte da infraestrutura que garante padrões mínimos de qualidade em nossos sistemas educacionais.

Nesse contexto, a formação de novos profissionais tem sido influenciada pela presença da tecnologia, ainda mais pelas condições de isolamento impostas pela pandemia de COVID-19. Assim, hoje, a formação dos futuros comunicadores sociais exige que eles não apenas dominem as técnicas de redação dos diferentes gêneros noticiosos, mas também aproveitem os recursos da Internet e programas de computador que facilitem a apresentação de dados, gráficos, tabelas, conhecendo a linguagem dos meios de comunicação e as técnicas de colocação de mensagens nos diferentes ambientes de comunicação e redes sociais. Adicionalmente, planear e realizar projetos de comunicação de outra natureza para serem aplicados em diferentes contextos e populações.

Este artigo mostra o mundo de possibilidades oferecido pela internet para a formação de comunicadores sociais que, por sua vez, formaram diferentes populações do território nacional durante o primeiro ano da pandemia enquanto realizavam seus estudos à distância.

Palavras-chave: Comunicação Educacional; Educomunicação; comunicação mediada; materiais multimédia; vídeos tutoriais 


\section{Introducción}

Cusipuma Arteaga Rosa

La pandemia de la COVID-19 ha originado una serie de consecuencias negativas en el mundo, en particular en las universidades de la región de América Latina, en donde las consecuencias permiten identificar, por el momento, tres elementos en la comprensión del escenario: una pérdida de los aprendizajes que bordea el $15 \%$ de pérdidas competencias, una pérdida de estudiantes que va del 3\% al 10\% en la región reportándose en algunos lugares hasta el 30\% menos de estudiantes matriculados y; una pérdida de la equidad (Instituto Internacional para la Educación Superior en América Latina y el Caribe [IESALC], 2021)

Las condiciones de encierro y la realización de actividades laborales en modalidad remota han afectado también las actividades académicas de la educación básica, la educación superior o terciaria que comprende a las universidades. Los criterios de calidad de algunas carreras, como las de Comunicación Social requieren que los estudiantes apliquen los conocimientos adquiridos en la práctica de campo, previo al ejercicio profesional. En ese contexto, el estudiante pone a prueba la utilidad de los conocimientos aprendidos para resolver problemas, así como vivir un proceso de socialización en donde identifique la presencia de factores culturales e interculturales, por ejemplo; antes de incorporarse a la vida activa en el mercado laboral. El ejercicio de esta práctica que pone a prueba la calidad de la formación académica son las que se han visto afectadas por las condiciones de aislamiento debido a la pandemia.

Para el estudiante de la especialidad de Comunicación Organizacional de la Universidad Nacional Mayor de San Marcos [UNMSM], es indispensable tomar contacto con poblaciones que les faciliten la comprensión del funcionamiento y desarrollo de las organizaciones, así como del papel que cada individuo aporta positiva o negativamente para el desarrollo de éstas; ya sean de base, populares, institucionales, empresariales o corporativas. El objetivo de estos encuentros, como en el caso del curso Comunicación Educativa, será tomar el pulso de la población, para que sobre la base del conocimiento producto de la investigación que forma parte de su proyecto en el curso-, los estudiantes propongan estrategias de comunicación que ayuden a la organización al logro de sus objetivos.

En el año 2020, primer año de la pandemia, las clases se realizaron en la modalidad remota. La incertidumbre y el temor a fallar en el intento de satisfacer los objetivos del curso dieron paso a la inteligencia y perspicacia de la juventud sanmarquina para enfrentar 
Cusipuma Arteaga Rosa

el reto. Al hacerse las clases en modalidad remota, también lo fueron las intervenciones que realizaron los estudiantes. Es objetivo de este artículo, analizar los resultados de sus intervenciones para el planteamiento de mejoras.

\section{¿Cómo entendemos la comunicación?}

Mario Kaplún (1985) en su texto El comunicador popular, diferencia la comunicación dominadora de la comunicación democrática, tomando como referencia la situación socio-política de la región en la segunda mitad del Siglo XX. De acuerdo con esa visión, los hombres y mujeres de América Latina sentían la necesidad de hacer escuchar sus voces, participar y ser los actores en la construcción de una sociedad más democrática. Era importante acceder a los medios masivos de comunicación. Por aquel entonces el modelo de comunicación de los mass media era sinónimo de transmisión de mensajes y nada más.

Las poblaciones rurales e indígenas de América Latina y por otro lado, las organizaciones de campesinos y mineros comenzaron a organizarse para generar sus propios contenidos radiales informativos, a diferencia de la propuesta de la comunicación de apoyo al desarrollo impulsado por Wilbur Schramm con el apoyo de la UNESCO. La experiencia de una larga cadena de estaciones conglomeradas y apoyadas por la Asociación Latinoamericana de Escuelas Radiofónicas [ERBOL] y la participación de quienes nada tienen que perder, le valió a Luis Ramiro Beltrán (2006) esbozar su primera definición de comunicación "La comunicación es el proceso de interacción social democrática que se basa sobre el intercambio de símbolos por los cuales los seres humanos comparten voluntariamente sus experiencias bajo condiciones de acceso libre e igualitario, dialógico y participación" (p. 65).

La definición que para efectos del curso, más se adecua al trabajo que realizan los estudiantes en estos menesteres, fue planteada por el mismo autor con posterioridad a la primera citada en el párrafo anterior:

La comunicación alternativa para el desarrollo democrático es la expansión y el equilibrio en el acceso de la gente al proceso de comunicación y en su participación en el mismo empleando los medios -masivos, interpersonales y mixtos- para asegurar, además del avance tecnológico y del bienestar material, la justicia social, la libertad para todos y el gobierno de la mayoría. (Beltrán, 2006, p. 66) 
En este entender de la comunicación han venido trabajando organizaciones reconocidas hasta la fecha como la Asociación Latinoamericana de Escuelas Radiofónicas [ALER], la Asociación Latinoamericana de Investigadores de la Comunicación [ALAIC], el Centro Internacional de Estudios Superiores de Comunicación para América Latina [CIESPAL], la Federación Latinoamericana de Facultades de Comunicación [FELAFACS], el Instituto para América Latina [IPAL] y muchas otras.

La irrupción de las tecnologías y la digitalización de la comunicación han llevado a la concentración de los medios en muy pocas manos y, hay una ausencia notoria, en la región de la radio y la televisión de servicio público. Son muy pocos los países en la región que cuentan con una estación o Red Pública. La experiencia mexicana cuenta con una Red Satelital de Televisión Educativa [EDUSAT] que es un sistema de señal digital comprimida que transmite de manera diaria a través de 16 canales de televisión:

Nueve de ellos administrados por Televisión Educativa de los cuales siete cuentan con programación propia $(11,12,14,17,21,24$ y 27) y uno para transmisiones especiales (26); el Instituto Latinoamericano de Comunicación Educativa [ILCE] tiene a su cargo los canales 13, 15, 16 y 18; el canal 23 está asignado al Consejo Nacional parala Cultura y las Artea [CONACULTA] y los canales 25 y 28 al Canal de Congreso y TV UNAM respectivamente. (SID Sistema Integrado de Documentación, 2021)

En otros países, a lo más que se llega es a contar con servicios de un canal de televisión pública destinado a la educación principalmente y en otros como en el caso del Perú, sólo una estación gubernamental o estatal de radio y otra para televisión. De otro lado, la información en los nuevos entornos y en los medios tradicionales de comunicación se discute como un "hecho de mercado" y no como "un hecho cultural y social”. (Beltrán, 2006, 73) De los más de 7,83 millones de personas en el mundo, más de 4,66 millones son usuarios de internet y 3,17 millones de personas permanecen desconectadas. Ellos representan "más del 40\% de la población mundial" (Hootsuite, 2021). Del total de usuarios de internet, la población que vive en el norte y occidente europeo y Norte América son los que cuentan con mayor cantidad de personas conectadas: 96\%, 93\% y 90\%, respectivamente (Hoootsuite, 2021).

La crítica desde las escuelas latinoamericanas de Comunicación Social pasan por hacer más válida la posición de no renuncia frente al statu quo caracterizado por la adscripción al mercantilismo y al tecnologismo de un sistema económico y social 
predominentemente neoliberal y globalizante. Democratizar la comunicación es una necesidad, siendo conscientes

\section{¿Qué es Comnicación Educativa?}

Aunque son muchos los autores que hoy en día identifican a la comunicación educativa o educomunicación como acciones educativas que se realizan dentro de las instalaciones educativas, en las relaciones de enseñanza-aprendizaje que se establecen entre docente y estudiante. Sin embargo, esta actividad, no tiene necesariamente que desarrollarse dentro del mundo escolar. Hasta antes de la pandemia, los estudiantes realizaban visitas a los asentamientos humanos, colegios, organizaciones de base, etc., para la realización de la parte práctica del curso: Tomar conocimiento de la organización, analizarla y discutirla desde el campo de la comunicación y recomendar estrategias para su solución.

¿Existe la posibilidad de cumplir estos objetivos, realizando las mismas actividades en los nuevos entornos mediáticos? La experiencia viene demostrando que sí, pero en casos limitados. El ejercicio requiere de que todos los involucrados cuenten con el acceso a una $\mathrm{PC}$ o algún mecanismo de comunicación inteligente y acceso a internet que les permita estar "en línea". Y eso, en la realidad de las regiones del país no es posible. La pandemia se encargó de demostrar que no fue posible, que no es posible y que, por esa razón muchos niños y adolescentes no realizaron o abandonaron el año escolar 2020.

A pesar de que la universidad no ha logrado escapar a estar realidad, al menos en América Latina y el Caribe, en donde la pandemia ha influido en los estudiantes, los profesores y el personal no docente y "ha ralentizado las actividades de investigación y extensión" (Quinteiro Goris, 2021); la continuación de la vida académica en entornos virtuales, ha facilitado la realización de las "actividades de campo" en estos mismos entornos virtuales, lo que ha conducido rápidamente a los estudiantes a desarrollar competencias digitales para que, por un lado, planifiquen y produzcan materiales mediáticos con fines educativos; y por otro lado, acompañen y apoyen con estrategias de comunicación a las organizaciones locales para la mejora de sus objetivos comunes. A la producción de materiales mediáticos con fines educativos, los expertos la denomina como la versión tecnologista de la comunicación educativa y al apoyo con estrategias de comunicación a las organizaciones locales, la identifican como educomunicación (Narváez-Montoya, 2019). 
Esta utilización de los "medios de comunicación” para afianzar el logro de objetivos educativos ocurre en diferentes casos: como cuando al salir de una consulta médica se le regala a una joven un boletín impreso ilustrativo para detectar tempranamente un cáncer al mama, el boletín que recibe una joven madre de familia con imágenes de cómo mantener las manos limpias y preparar los alimentos para su bebé, también lo son los videos que las personas usuarias del servicio de un ministerio u oficina pública visualizan mientras esperan su turno de atención. A través de esos mensajes, se da a conocer los distintos servicios de la entidad, los trámites y sus costos, el tiempo de duración aproximado, entre otros. El objetivo de estos materiales es importante para su utilización, sin embargo, más importante para los estudiantes de Comunicación es conocer para qué se va a producir, qué se va a decir y cómo se va a decir.

La formación integral y humanista del estudiante de Comunicación Social ha permitido que a través de la realización de proyectos, en el marco de sus salidas a campo, establezcan relaciones de beneficio mutuo con las distintas poblaciones con las que han venido trabajando: estudiantes de los niveles primario y secundario, madres de familia, recicladores, pobladores de asentamientos humanos, madres adolescentes, comedores populares, grupos de migrantes, etc. Con todos ellos han trabajado un diagnóstico para conocer necesidades puntuales y comunes, lo que les ha valido para el desarrollo de sus propuestas. Todo realizado con la participación directa de la población, lo que les ha valido para comprender que es necesario "poner como base del proceso de enseñanza/aprendizaje la participación activa de los educandos" (Kaplún, 1997). Para el planteamiento de sus propuestas coordinan, gestionan, movilizan la participación de expertos, producen herramientas y materiales en donde las personas que participan de la acción educativa son sujetos activos de la educación. Participan en la construcción y recreación del conocimiento.

En este contexto, las acciones de enseñanza-aprendizaje se convierten en un diálogo en el que quienes asumen el rol de educandos aportan desde sus experiencias previas el enriquecimiento del diálogo. El resultado de esta praxis confirma que el aprendizaje de acuerdo con Vygotsky, es un producto social en donde "Aprendemos de los otros y con los otros" (Kaplún, Procesos educativos y canales de comunicación, 1998), el rol de los educandos entonces, no solamente es "aprender", también intercambian información, comparten experiencias, los mayores “enseñan” a los más jóvenes y los más jóvenes "facilitan" la comprensión de nuevos términos a los mayores o les ayudan a "operar" los 
equipos con mayor destreza y agilidad.

Los diferentes casos en los que se desarrolla la comunicación educativa van más allá de la aplicación de materiales auxiliares e instrumentales ha permitido a los estudiantes de Comnicación Educativa adentrarse en conocimiento de la diversidad cultural y social de los pobladores de Lima. Estas relaciones les ha facilitado el desarrollo de habilidades para el diálogo, la comprensión de distintos enfoques frente a los problemas de la ciudad, entre otros, y lo más importante, la posibilidad de presentar mediante propuestas que son discutidas con la comunidad, las vías de solución a sus problemas con estrategias de comunicación, planificadas y organizadas. Las diferencias intergeneracionales, multiculturales, educativos, sociales, económicos entre los tantos que nos separan, desaparecen para dar paso a la comunión de ideas para dar solución a los problemas.

\section{Entornos de aplicación}

Hasta el año 2019, las distintas agrupaciones de ciudadanos o estudiantes, así como organizaciones en donde se desarrollaron los proyectos de los estudiantes de Comunicación Educativa fueron los distintos distritos de la ciudad de Lima Metropolitana. Con la finalidad de tener información que le brinde sostenibilidad a sus hallazgos, los estudiantes consultaban la data del Instituto Nacional de Estadística e Informática [INEI], así como las distintas encuestas de los ministerios y organizaciones de la sociedad civil. De acuerdo con el décimo informe urbano de percepciones sobre la calidad de vida en la ciudad, elaborada por Lima Cómo Vamos (2019), se tenía:

- El 37.5\% de personas que vive en la capital se siente satisfecho con Lima como una ciudad para vivir, al mismo tiempo que un $21.7 \%$ se siente insatisfecho y un $40.8 \%$ se coloca en punto medio.

-Entre las preocupación más sentidas por el ciudadano común se afirma que el $82.2 \%$ de las personas siente que la inseguridad ciudadana es importante y afecta la calidad de vida en la ciudad, el $46.2 \%$ considera al transporte público como el segundo gran problema y, el 38.6\% considera que la corrupción de funcionarios o servidores públicos es el tercer gran problema de la ciudad.

- El tema de la situación del medio ambiente preocupa a los limeños o quienes viven en Lima. Así, ubican como un factor que afecta el ambiente a la contaminación por vehícular en un $72.3 \%$, la falta de árboles y mantenimiento de la zonas verdes en un $40.8 \%$ 
y el sistema de recojo de basura en un $35.8 \%$.

- Con relación a la satisfacción con el espacio público disponible en la ciudad mientras el $30.7 \%$ estima que se encuentra insatisfecho, el $23.8 \%$ se siente satisfecho y el $45.4 \%$ regular.

- Con relación a las ofertas de actividades reccreativas y culturales en Lima el $40.2 \%$ se siente insatisfecho, el $35.7 \%$ ni satisfecho, ni insatisfecho y el $22.8 \%$ satisfecho.

Se contaba con información sobre la población de Lima capital que había sufrido un proceso de migración interna muy fuerte desde los años 80 's del siglo XX, con sujetos que se habían incorporado aportando su cultura y aspectos culturales tradicionales, que había tenido incidencia en la falta de puestos de trabajo y que muchos, en particular los que provenían de poblacionies rurales se habían asentado en las laderas de los cerros, en barriadas cumpliendo tareas de comercialización en mercados y mercadillos, reciclamiento de basura o trabajaban en fábricas informales.

La irrupción de la pandemia en el año 2020 brindó la oportunidad a los estudiantes para abrirse al conocimiento de poblaciones distintas a las de la gran ciudad, con necesidades y problemas distintos. Aprendieron sobre las condiciones de vida que tienen los estudianes que asisten a una escuela pública en el centro poblado de Chachapoyas, en el departamente de Amazonas, o los que asisten a una escuela en asentamiento humano Simón Bolívar en el departamento de Arequipa, o los pobladores del centro poblado de San Francisco, en Querocotillo, en el departamento de Piura, entre otros. La pandemia permitió conocer las inmensas posibilidades de la digitalización de la comunicación, para la realización de encuestas, entrevistas, actividades de capacitación, y mucho más. Los estudiantes fueron capaces de comprender que sí es posible hacerlo, pero, para lograrlo el acceso al ciberespacio es condición fundamental.

\section{Comunicadores en acción}

Los estudiantes de Comunicación Educativa se dedican a indagar entre las diversas organizaciones que existen en sus localidades o sus cercanías las posibilidades de acceder con los pobladores para realizar un trabajo de investigación que culmine con una propuesta de estrategia de comunicación en la que el beneficio resulte para investigadores e investigados. Una vez que son aceptados, los estudiantes inician el trabajo de análisis situacional y delimitan los ambientes internos, microambientes y macroambientes de la comunicación en la organización. Para ello, solicitan a los integrantes de la comunidad o 
asociación, la mayor seriedad en la data que van a conseguir, porque de ello dependerá el éxito de su participación. Normalmente, esto se realiza mediante un oficio o carta de presentación por parte de la Universidad para darle las seguridades al estudio.

Posteriormente, se da el proceso de investigación que culmina con la realización del diagnóstico que además comprende la propuesta de estrategias y objetivos de comunicación, la elaboración de mensajes y de materiales comunicativo-informacionales y educativos, así como la posibilidad de realizar actividades transferencia de conocimientos para retroalimentar a la población. El proceso termina con una evaluación general de la participación de la población. A lo largo de todo el semestre, la responsabilidad de los estudiantes evidenciada semanalmente frente a la población hace que su participación sea reconocida y valorada aún con posterioridad a la realización y culminación del proyecto.

Para el proceso de transferencia de conocimientos, debido a que algunos problemas detectados y seleccionados con la población, son de ámbitos distintos a la comunicación; los estudiantes se ven en la imperiosa necesidad de realizar tareas de advocacy y coordinación para gestionar en las otras Escuelas Profesionales de la Universidad, la ayuda profesional que les permita el acceso a información puntual, contar con la participación de expertos y muchas veces, la realización de campañas de salud, como las logradas en años anteriores con las Facultades de Medicina Humana, Odontología, Farmacia y Bioquímica, Medicina Veterinaria, Psicología o la Unidad de Medicina Tropical, entre otros y también la posibilidad de contar con la intervención y el respaldo de algunas empresas e industrias y, organizaciones públicas y privadas.

Una vez llegada a esta etapa, los estudiantes sanmarquinos redoblan esfuerzos para la producción de materiales educativo-informativo-comunicacionales de acuerdo a las necesidades de los usuarios, entre muchos aspectos que deben cuidar, cada estudiante procura:

-Que sus mensajes gocen de legitimidad, para ello, los estudiantes de comunicación están en la obligación de conocer los temas que los expertos van a desarrollar con la población, que requiere mayor explicación.

-Que sus mensajes sean competentes, la posibilidad de acceder a material informativo brindado por el personal experto con la finalidad de producir materiales de divulgación facilita que sus mensajes sean tomados en cuenta. La competencia del 
mensaje resulta producto de la validación técnica del material de ser entregado a los participantes.

-Que sus mensajes sean persuasivos, además de brindar información los materiales producidos por los estudiantes movilizan emociones o invitan a transformar una actitud.

-Que sus mensajes sean apropiados, no solo por el contenido del material, otros factores que entran en juego son las dimensiones, los colores, el tamaño de las letras, la presentación, el uso que se le va a otorgar, etc. (Niño, 2011)

Todos los procesos desarrollados en el marco del proyecto de intervención se realizan con el conocimiento y participación de la población involucrada. Para ello, semanalmente, los estudiantes apelan a distintas estrategias didácticas que facilitan la participación activa de los asistentes, a fin de que puedan construir y reconstruir sus conocimientos, habilidades y actitudes. Entre las estrategias didácticas de mayor uso en estas actividades se han encontrado el aprendizaje basado en problemas (ABP), juego de roles, trabajos prácticos, experiencias exitosas, talleres, visionados, entre otros que debido a la organización y planificación rígida les asegura la validez de su intervención (MINSA, 2016)

De acuerdo con Kimberly y Nentley (1999) citado en Niño para afrontar el futuro los ciudadanos deben desarrollar seis habilidades básicas que derivan de la creatividad:

- Habilidad para la gestión de la información, que consiste en la capacidad que tienen las personas para seleccionar, valorar, organizar y aplicar la información en el marco de la profusa cantidad de información a los que los seres humanos se encuentran expuestos.

- Habilidad para la auto-organización, necesaria para definir metas, planificar actividades en el tiempo, asumir responsabilidades, determinar recursos y aplicar las mejores estrategias para el conocimiento.

- Capacidad para la interdisciplinariedad, se hace necesario afrontar el conocimiento y la misma solución de problemas desde diferentes miradas, saberes y disciplinas

- Habilidades personales e interpersonales, es necesario que la persona se conozca a sí misma profundamente, cuente con la motivación y sea capaz de manejar buenas relaciones interpersonales. 
- Habilidad para la reflexión, la evaluación y la autoevaluación; esto exige, entre otras, la capacidad para observar, analizar, valorar, emitir juicios equilibrados, sopesar opciones, tomar decisiones y ajustarse a la realidad, según el contexto.

- Asumir la gestión del riesgo los riesgos varían de una persona a otra, de una sociedad a otra y, en general, de un contexto a otro. Además, los riesgos se dan a muy diferentes niveles: en el conocimiento, en las conductas y procedimientos, vida personal (saludo, empleo.), desempeño social, etc. (Niño, 2011)

\section{¿A quiénes se beneficia?}

Una revisión de los proyectos da cuenta de los temas desarrollados, sus objetivos y las actividades realizadas por los futuros comunicadores sociales:

- Creación de estrategias de comunicación para la elaboración de material educativo dirigido a estudiantes del $4^{\circ}$ grado del nivel secundario de la Institución Educativa Emblemática "San Juan de la Libertad" de Chachapoyas

Los estudiantes sanmarquinos aunaron esfuerzos con la finalidad de contribuir a la gestión del aprendizaje y reducir las brechas educativas por la presencia del COVID-19. En este contexto, produjeron materiales multimediales dirigido a los alumnos que reforzaron lo brindado en el programa "Aprendo en casa" y, sumaron a acciones de capacitación dirigida a los docentes para mejorar la calidad del proceso enseñanzaaprendizaje y el uso de herramientas digitales.

Los alumnos (15varones y 17 mujeres) que se beneficiaron con la intervención del grupo de estudiantes sanmarquinos fueron los del 4to. grado, del aula 26 "Abraham Valdelomar" de la Institución Educativa Emblemática "San Juan de la Libertad", de Chachapoyas, región Amazonas. Del total de estudiantes, 25 viven en la zona urbana y 7 en la zona rural.

A lo largo de las doce (12) semanas de duración del proyecto se produjeron materiales educativo comunicacionales, en el marco de los contenidos de la malla curricular nacional que se caracterizaron por poseer un tono formal y dinámico, con una redacción simple, de léxico comprensible y no complicado. De manera similar se procedió para la elaboración de los materiales de audio. 
- Ciudadanía Digital

Cusipuma Arteaga Rosa

Descubrir que existen muchas escuelas en el país en donde los docentes no cuentan con un conocimiento sobre el uso de las TIC para la realización de sus clases vía internet y que en muchos casos, no se encuentran motivados para hacerles llegar materiales sobre los diferentes temas de sus cursos, impulsó a los estudiantes de Comunicación Educativa plantearse el proyecto sobre Ciudadanía Digital para ser desarrollado en la Institución Educativa 40175 Gran Libertador Simón Bolívar ubicado en el asentamiento humano Simón Bolívar del distrito de José Luis Bustamante y Rivero del Departamento de Arequipa.

Desde el inicio de las clases, los docentes sólo enviaban publicaciones en el WhatsApp y la recepción de mensajes por parte de los estudiantes. Producto de la realización de una encuesta se determinó la necesidad de los estudiantes para acceder a conocimientos sobre Historia del Perú, Ciudadanía y, Espacio Geográfico, Sociedad y Economía.

El proyecto de Comunicación Educativa estuvo destinado a los alumnos del 5to. "A", conformado por 24 alumnas y 10 alumnos. De este total solo 24 alumnos tienen acceso al programa Aprendo en Casa y 10 no. Como producto de los cuestionarios aplicados para diagnosticar la realidad de los alumnos se establecieron entre los objetivos de comunicación: mejorar el conocimiento sobre los valores y funciones que tienen las instituciones gubernamentales como el Ministerio de Economía y Finanzas, el Ministerio del Ambiente y el Ministerio de Cultura, concientizar sobre la importancia de la participación ciudadana y cómo ésta posibilita el cambio dentro de la sociedad, enre otros.

En su estrategia de acercamiento y abordaje de los temas, los estudiantes de la carrera de Comunicación Social tomaron contacto con los alumnos mediante convocatoria para el concurso "Ciudadanía Digital" que les facilitó la participación general de los estudiantes del aula. Entre las piezas producidas para el proyecto se tienen Guías de orientación para el uso de los portales de los Ministerios mencionados líneas arriba, podcast, infografias. El acceder a información despertó el interés de los estudiantes para indagar en la web distintos repositorios y plataformas informativas.

- Mejoramiento del uso y manejo de las TIC en el proceso de enseñanzaaprendizaje del curso de Desarrollo Personal, Ciudadanía y Cívica

En el marco del confinamiento debido a la pandemia originada por el COVID-19, 
los estudiantes de Comunicación Educativa contactaron con la docente del curso Desarrollo Personal, Ciudadanía y Cívica del Colegio "José Carlos Mariátegui" con la finalidad de promover y capacitar en el uso y aplicación de las TIC como complemento en el proceso enseñanza-aprendizaje de los alumnos del 5to. "E”. En este contexto y como producto de las coordinaciones establecidas, el objetivo del proyecto estuvo destinado a capacitar a la docente para que con un mejor desempeño en el conocimiento y aplicación de las tecnologías pueda lograr un mejor desempeño en la enseñanza de su asignatura. Toda vez que los alumnos experimentaban que iban a perder el año debido a que no recibían clases en el curso y mucho menos materiales que les pudieran servir de referencia para el desarrollo del curso.

Con este propósito una vez identificados los beneficios que traería la capacitación en la docente para hacer un uso adecuado de las TIC en la organización de sus sesiones de clase y por ende, el beneficio directo en los alumnos de su curso, se procedió a establecer los objetivos educativos de la intervención, así como la organización de todas las actividades a desarrollar con la docente, teniendo como medio de comunicación el WhatsApp y correo electrónico.

En el marco de los materiales producidos para capacitar a la docente se tienen un video tutorial de Canva, videos de capacitación para retroalimentación, realizacion de una infografía y presentaciones con las que capture la atención de los alumnos. Durante las doce (12) semanas de interacción con la docente se consolidó el mensaje: "Las TIC no hacen un excelente profesor, pero un excelente profesor sí emplea bien las TIC". La evaluación final de lo realizado en el proyecto arrojó como resultado que se lograron los comportamientos deseados en la docente.

- Mejoramiento de las estrategias de enseñanza y las oportunidades de aprendizaje mediante el uso de recursos tecnológios y la ampliación de herramientas pedagógicas en el aula del 5to. D de secundaria de la I.E. 3037 República de Canadá

La intervención realizada por los estudiantes de Comunicación Educativa mediante este proyecto, tuvo como objetivo principal la mejora del proceso de aprendizaje de los estudiantes del curso de Arte, del 5to. año "D” del Colegio Rapública de Canadá del distrito de Comas que consistió en brindarles estrategias que faciliten la interacción pedagógica para propiciar la innovación y creatividad en la comunicación mediante productos comunicacionales eficaces, fomentar el uso de las TIC para impulsar el aprendizaje y elaborar recursos educativos que aporten al desarrollo de las clases y un 
aprendizaje óptimo.

Cusipuma Arteaga Rosa

De acuerdo con la estrategia diseñada por los estudiantes, se recurrió a acciones de capacitación dirigidas a la docente para un mejor uso de las herramientas digitales: Google Meet, Google Drive y Canva con las que se buscó mejorar la interactividad con los estudiantes, contar con un repositorio de trabajos de sus alumnos e introducirlos en el uso de las herramientas tecnológicas para el diseño y propuestas de diseño artístico. En el ámbito de las producciones de audio, se realizó la capacitación para la creación de un spot educativo con Audition que facilitó la comprensión de conceptos y nociones básicas de Adobe Audition y el empleo de este programa para retroalimentar sus conocimientos previos y un mejor desempeño frente a sus estudiantes.

Para los 29 estudiantes del 5to. Año "D" se realizó el taller "Inhalo paz, exhalo estrés" para que los alumnos puedan manjar el estrés con técnicas para gestionar situaciones de estrés en su vida cotidiana, interiorizar la técnica de relajaación progresiva de Jacobson y reconocer las actividades que les ayudan a gestionar su tiempo.

\section{- Lomas, un futuro mejor para todos}

El objetivo principal del proyecto fue informar sobre la sostenibilidad ambiental de las Lomas El Mirador del distrito de San Juan de Lurigancho, el impacto ambiental negativo que se ejerce con las distintas actividades de la población circundate y la necesidad de generar un impacto positivo en el lugar. Las acciones están dirigidas a la población de mujeres, amas de casa, que se dedican a la atención de su hogar, cuyas edades oscilan entre los 30 y 44 años de edad, del Asentamiento Humano Nuevo Perú y que viven en los alrededores de las Lomas El Mirador. El proyecto incluyó visitas de los estudiantes a la zona para realizar el diagnóstico y determinar el conocimiento que las amas de casa tenían con relación a las Lomas El Mirador.

A lo largo de diez (10) fechas, en modalidad remota, los estudiantes de Comunicación Educativa organizaron una campaña de información dirigida a las amas de casa con quienes se compartió materiales para invitarlas a participar de la capacitación, así como el envío de materiales con los cuales se realizó el reforzamiento de los objetivos de comunicación y una actividad de capacitación en el que se compartió una canción creada para la campaña y se realizó la distribución de los materiales multimediales, producidos por los estudiantes.

A través de fichas de evaluación, los estudiantes relizaron el monitoreo 
correspondiente para el cumplimiento de los objetivos de comunicación del proyecto.

- Sal de mi Tierra: Proyecto de sensibilización sobre el impacto negativo de la salinización en la actividad agrícola realizado para el nivel secundario del colegio San Francisco de Chocán del distrito de Querecotillo, Valle de Chira

El proyecto tuvo por objetivo principal: Informar, visibilizar y sensibilizar sobre la problemática de la salinización, y el papel que tiene la actividad humana dentro de ella, a los alumnos de educación secundaria de la I.E. San Francisco de Chocán en el distrito de Querecotillo.

Entre sus objetivos secundarios se tenían el dar a conocer sobre las causas de la salinización y la situación de la salinización en el distrito de Querecotillo, asi como los métodos para combatir la salinidad; diseñar y producir recursos audiovisuales informativos sobre este problema así como promover en los estudiantes el acceso a la información sobre el tema.

El proyecto consistió en elaborar una campaña educativa-informativa para enseñar a los estudiantes el concepto de la salinización y que este problema afecta las tierras en el distrito de Querecotillo con repercusiones negativas en los campos de cultivo y consecuentemente las actividades económicas y productivas del distrito.

Para la distribución de los mensajes y organización de la campaña, se hizo uso de los siguientes canales de difusión: Whatsapp, Facebook y Soundcloud.

\section{Asegurando la calidad de la formación de los comunicadores}

El desarrollo de estos proyectos contribuye con el logro del sétimo objetivo de calidad de esta Casa de Estudios que tiene por finalidad fortalecer la interrelación universidad-sociedad "basada en el intercambio de conocimientos y experiencias para el desarrollo integral conjunto, respondiendo y anticipándose a las necesidades de la sociedad" (Universidad Nacional Mayor de San Marcos [UNMSM], 2020). La comunicación establecida entre los estudiantes de Comunicación con las distintas poblaciones del país rompe con la reflexión individual y limitada de la sociedad, facilita la comunicación, el diálogo intercultural y los introduce en la comprensión holística del mundo, preparándolos para un ejercicio profesional pertinente, ético y consciente de que en el futuro serán los encargados de propiciar los espacios para un diálogo democrático e intercultural. 
La responsabilidad de la formación de los jóvenes comunicadores sociales se da en el marco de compender que la educación no solo es un derecho, sino que; la educación con calidad es un derecho (UNMSM, 2020, 32), de acuerdo con la Ley General de Educación [LGE], Ley 28044 que define a la calidad educativa como "el nivel óptimo de formación que deben alcanzar las personas para enfrentar los retos del desarrollo humano, ejercer su ciudadanía y continuer aprendiendo durante toda la vida". En esta misma línea de comprensión de la calidad educativa los jóvenes, cuyo trabajo se ha destacado en este artículo, se realiza en el contexto de comprender la calidad de la educación universitaria como "la coherencia del ser y quehacer con el deber ser institucional en el desarrollo integral y permanente de la misión, la visión y las funciones de la universidad, con ética y pertinencia para responder a las necesidades de la sociedad" (UNMSM, 2020, 21). La preparación de los jóvenes comunicadores, no solamente tiene que darse en el contexto del aula, se realiza también en el ejercicio de lo que será su quehacer profesional, desarrollando capacidades y habilidades que modelen su actuar competente ético, científico y técnico, capaces de liderar la resolución de problemas del país.

\section{Reflexión final}

En muchos espacios académicos en donde se reflexiona sobre las condiciones en las que hoy se realizan las clases se escucha que las tecnologías llegaron para quedarse. Pero no todos los estudiantes están en condiciones de recibir en condiciones óptimas las clases por internet. Las tecnologías cambiaron en el trabajo docente la manera en que se realizan las coordinaciones, la la organización de los contenidos, la manera y los tiempos en que son compartidos con los estudiantes. Cambió la manera en que se realiza la evaluación, los trabajos grupales, etc. El uso de estas tecnologías demanda una buena calidad de internet y los estudiantes comparten las computadoras en casa y esperan su turno, pierden la conexión cuando reciben una llamada telefónica, etc. A pesar de los esfuerzos que realizan, no pueden escapar a factores como la falta de energía eléctrica y muchos otros que ponen en riesgo el logro de los objetivos de su formación que no se pueden soslayar.

Como consecuencia de lo avanzado hasta el momento, se planean algunas consideraciones que podrían ayudar a plantear soluciones urgentes:

- Aumento en el número de laboratorios para el diseño y producción de materiales 
multimediales.

- Implementación de laboratorios exclusivos para realizar trabajos de investigación científica con programas de análisis.

- Involucramiento de las Oficinas de Responsabilidad Social de la Universidad para el seguimiento de los proyectos ejecutados por los estudiantes.

- Realización de convenios con organizaciones de la sociedad civil que faciliten el acceso de los estudiantes para la ejecución de sus proyectos de comunicación.

\section{Referencia bibliográfica}

Bello, M. (octubre de 2018). Los COAR: la persistencia de un error. Revista Tarea. Lima, Perú.

Beltrán, L. R. (enero-junio de 2006). Anagramas Rumbos y Sentidos de la Comunicación. La Comunicación para el Desarrollo en Latinoamérica: Un recuento de medio siglo. Medellín, Colomia.

Escarpit, R. (1977). Teoría General de la Información y la Comunicación. Barcelona: Icaria.

Hootsuite. (enero de 2021). We are social Hootsuite. Digital 2021 Global Overview Report.

Hootsuite. (January de 2021). Hootsuite. Obtenido de Digital 2021 Global Overview Report: https://hootsuite.widen.net/s/zcdrtxwczn/digital2021_globalreport_en

Instituto Internacional para la Educación Superior en América Latina y el Caribe [IESALC]. (24 de enero de 2021). Obtenido de Instituto Internacional para la Educación Superior en América Latina y el Caribe IESALC: https://www.iesalc.unesco.org/2021/01/24/la-transformacion-digital-durante-lapandemia-de-la-covid-19-y-los-efectos-sobre-la-docencia/\#.YLa6nJMzZN1

Instituto Internacional para la Educación Superior en América Latina y el Caribe [IESALC]. (24 de enero de 2021). Obtenido de Instituto Internacional para la Educación Superior en América Latina y el Caribe IESALC: https://www.iesalc.unesco.org/2021/01/24/la-transformacion-digital-durante-lapandemia-de-la-covid-19-y-los-efectos-sobre-la-docencia/\#.YLa6nJMzZN1 
Kaplún, M. (junio de 1997). De medios y fines en comunicación educativa. Revista Latinoamericana de Comunicación Chasqui(58), 4-6.

Kaplún, M. (1998). Procesos educativos y canales de comunicación. Comunicar(11), $158-165$.

Kaplún, M. (1998). Procesos educativos y canales de comunicación. Comunicar(11), 158-165.

Lima como vamos, Lima y Callao según sus ciudadanos. Décimo Informe Urbano de Percepción sobre Calidad de Vida en la Ciudad 2019 https://www.limacomovamos.org/wp-content/uploads/2019/11/Encuesta$\underline{2019 . p d f}$

Narváez-Montoya, A. (11 de marzo de 2019). Comunicación educativa, educomunicación y educación Mediática: una propuesta de investigación y formación desde un enfoque culturalista.

Niño Rojas, V. M. (2011). Competencias en la comunicación. Hacia las prácticas del discurso. Bogotá, Colombia: OCOE.

Quinteiro Goris, J. A. (1 de junio de 2021). Instituto Internacional de la UNESCO para la Educación Superior en América Latina y el Caribe. Recuperado el junio de 2021, de COVID-19: su impacto en la Educación Superior en los ODS: https://www.iesalc.unesco.org/2021/06/01/covid-19-su-impacto-en-la-educacionsuperior-y-en-los-ods/\#.YL2H7DYzZN2

UNMSM (2020) Política de Educación con Calidad y Sistema de Gestión de Calidad Educativa de San Marcos. Oficina Central de Calidad Académica y Acreditación. Lima, Perú. 74 pp.

Sistema Nacional de Evaluación, Acreditación y Certificación de la Calidad Educativa [SINEACE] (2017) Modelo de Acreditación Institucional para Universidades. Lima, Perú. 36 pp. 